\title{
Alianza terapéutica y adherencia a tratamientos de drogodependencia: un estudio en usuarios de centros de atención de la intercomuna Concepción - Talcahuano*
}

\author{
David Jesús Vidal Corominas** \\ Isis Chamblás García***
}

\section{RESUMEN $^{1}$}

La investigación sobre los factores asociados a la adherencia a los tratamientos es considerada un área prioritaria en las ciencias de la salud (Pereria, 2009). Determinar cuáles son los factores que influyen en una buena adherencia, juega un papel crucial en la efectividad de la terapia utilizada (Hernández et al., 2009). El presente estudio se plantea la necesidad de dimensionar el efecto que la alianza terapéutica ejerce sobre la adherencia en pacientes con problemas de drogodependencia, y establecer cuáles otras variables o condiciones permiten que este componente del proceso terapéutico se establezca y sirva de puente en la relación entre el profesional de la salud y el usuario. Los resultados indican elevada alianza terapéutica entre usuarios y profesional, consistencia en sus evaluaciones en alianza total y por componentes, así como significación estadística entre la alianza terapéutica y sus respectivas subescalas con adherencia a tratamiento.

Palabras clave: alianza terapéutica, drogodependencias, adherencia a tratamiento.

\section{Aliança terapêutica e aderência aos tratamentos para dependência de drogas: um estudo sobre os usuários dos centros da intercomuna Conceição - Talcahuano}

RESUMO

A pesquisa sobre fatores associados à adesão aos tratamentos é considerada uma área prioritária nas ciências da saúde (Pereria, 2009).

Artículo recibido: 03/12/2013. Artículo aprobado: 13/08/2014. Versión final: 08/09/2014.

** Chileno. Psicólogo. Magíster en Trabajo Social y Políticas sociales, Universidad de Concepción. Dirección de Educación, Municipalidad San Pedro de la Paz, Concepción (Chile). Correo electrónico: davidalc09@live.com

*** Chilena. Trabajadora Social, Magíster en Educación para el Trabajo Social, Universidad Católica de América, Washington D.C. Profesora Asociada Departamento de Trabajo Social, Facultad de Ciencias Sociales, Universidad de Concepción, Chile. Correo Electrónico: ichambla@udec.cl

Este trabajo forma parte de la Tesis del investigador David Vidal para optar al Grado de Magíster en Trabajo Social y Políticas Sociales de la Universidad de Concepción. 
Estabelecer quais são os fatores que influenciam uma boa adesão, desempenha um papel crucial na eficácia da terapia utilizada (Hernández et al., 2009). O presente estudo manifesta a necessidade de medir o efeito que a aliança terapêutica exerce sobe a aderência em pacientes com problemas de toxico dependência e estabelecer quais outras variáveis ou condições permitem que este componente do processo terapêutico se estabeleça e sirva como uma ponte no relacionamento entre o usuário e o profissional de saúde. Os resultados indicam elevada aliança terapêutica entre usuários e profissionais, consistência em suas avaliações em aliança total e por componentes, bem como de significância estatística entre a aliança terapêutica e suas respectivas subescalas com adesão ao tratamento.

Palavras-chave: aliança terapêutica, toxico dependência, adesão ao tratamento.

\title{
Therapeutic partnership and adherence to drug dependence treatment: A study in users of Concepcion - Talcahuano inter-municipality clinics
}

\author{
ABSTRACT
}

\begin{abstract}
Research on factors associated with adherence to treatment is considered a priority in health sciences (Pereira, 2009). Identifying factors that influence good adherence plays a crucial role in the effectiveness of the therapy used (Hernandez et al., 2009). The present study addresses the need to assess the effects the therapeutic partnership has on adherence in patients in drug abuse situation and to establish what other variables or conditions allow this component of the therapeutic process and strengthen the relationship between the health provider and the user. The results show a strong therapeutic partnership among users and health providers, consistency in their assessments in full partnership and by components as well as statistical significance between the therapeutic partnership and their respective subscales with adherence to treatment.
\end{abstract}

Keywords: therapeutic partnership, drug dependence, adherence to treatment.

\section{Presentación}

La Organización Mundial de la Salud asegura que "el incumplimiento del tratamiento es la principal causa de que no se obtengan todos los beneficios que los tratamientos pueden proporcionar a los pacientes"2 (OMS). Este obstáculo en el tratamiento, en lo referido a que el usuario adopte un nuevo estilo de vida o una

http://www.who.int/mediacentre/news/releases/2003/pr54/es/ 
nueva forma de entender su realidad, contrasta con el avance en las últimas décadas en los tratamientos farmacológicos y psicológicos, algo que no se ha reflejado en el área de la adherencia (Pereira, 2009).

El interés por la adherencia al tratamiento y su relación con el vínculo que se genera entre los profesionales de la salud y sus usuarios, ha estado presente a lo largo de la historia y ha suscitado grandes interrogantes (Hernández et al., 2009.) Los estudios sobre adherencia que se han realizado en la última década señalan tasas de abandono que varían desde el 20\% hasta 60\% de los pacientes que acuden a recibir un tratamiento a centros de salud mental (Altamura y Malhi, 2000), pudiendo sobrepasar el $60 \%$ cuando se evalúa la no asistencia a la primera cita de toma de contacto (Bachman, 1993). En lo referido al tratamiento de la drogodependencia en Chile, la deserción de los tratamientos puede llegar al 70\% en la modalidad residencial y entre un 50\% y $60 \%$ en los otros tipos de tratamiento (CONACE, 2010).

El problema de la adherencia terapéutica se presenta siempre que se requiere auto-administrar el tratamiento, independientemente del tipo y la gravedad de la enfermedad y de la accesibilidad a los recursos de salud. Los factores que influyen en la adherencia son múltiples, algunos de estos factores se relacionan con el paciente, pero también influyen notoriamente las características de la enfermedad y su tratamiento, los atributos del sistema de asistencia sanitaria y la prestación de servicios (OMS, 2004).

La importancia del problema en el incumplimiento de los tratamientos se hace indiscutible si se analizan las repercusiones que éste tiene desde el punto de vista clínico, médico, económico y psicosocial, puesto que afecta cuestiones que tienen que ver con la calidad de la atención, con la relación médico-paciente, con el uso racional de recursos y servicios de salud, entre otros. De ahí que se convierta en un asunto serio para la salud pública contemporánea, más si se tiene en cuenta que es un problema mundial, que se presenta en todos los países con independencia de su nivel de desarrollo, y de alarmante magnitud, sobre todo en las regiones más pobres (OMS, 2004). En lo referido al tratamiento de la drogodependencia, la relación entre la eficacia del tratamiento y la adherencia ha sido abordada por diversos autores. Fue Vaillant (1966) uno de los primeros que contempló entre 
sus análisis esta relación, y fue precisamente él quien sugirió la existencia de una relación inversa entre la duración de la estancia de los pacientes en el Hospital Lexington de Nueva York y la recaída posterior. Según éste y mediante estudios diversos, ha confirmado esta hipótesis y ha sugerido que un indicador de buen pronóstico es la permanencia en el programa por encima de los tres meses.

El abandono y la poca adherencia lograda en las intervenciones sobre las adicciones se trasforman en un escollo a superar por parte de las instituciones y los equipos ligados al tratamiento de esta problemática. Encontrar las variables que refuerzan la permanencia del paciente, representaría un avance en torno a este tipo de tratamiento y, a la larga, disminuiría el gasto público asociado a este trastorno.

Es precisamente en este contexto que se hace necesario esclarecer cuáles son las variables asociadas a una buena adherencia al tratamiento y es aquí donde aparece con relevancia el término Alianza Terapéutica, concepto que más se ha asociado a la permanencia o a la adherencia de las intervenciones psicoterapéuticas (Solano, 2001). La alianza terapéutica (AT), en términos generales, fue usado para explicar la relación existente entre terapeuta y paciente durante el proceso psicoterapéutico (Hartley, 1985). En la década de 1970, Bordin (1976) presentó una definición transteórica, planteando que la alianza sería una relación consciente que involucra acuerdos y colaboración entre terapeuta y paciente, concepto que integra tres componentes: vínculo, tareas y metas, los que en combinación definirían la calidad y fuerza de toda alianza.

Aunque existe un cuerpo de investigaciones que respaldan la significación de la alianza terapéutica en el proceso de la psicoterapia, en el área de adicciones no se han realizado suficientes estudios que den cuenta de esta relación y, además, de su incidencia en la adherencia al tratamiento (Planes, 1991).

De ahí que el presente estudio se plantea dimensionar el efecto que la alianza terapéutica ejerce sobre la adherencia en pacientes con problemas de drogodependencia y establecer cuáles otras variables o condiciones permiten que este componente del proceso terapéutico se establezca y sirva de puente en la relación entre el profesional de la salud y el usuario. 
La investigación sobre los factores asociados a la adherencia a los tratamientos es considerada como un área prioritaria en las ciencias de la salud (Pereira et al., 2009). Determinar cuáles son los factores que influyen en una buena adherencia, juega un papel crucial en la efectividad de la terapia utilizada (Hernández et al., 2009).

En lo referido al tratamiento de las adicciones, se hace de suma importancia estudiar los factores asociados a la adherencia, debido a que gran parte de las indicaciones terapéuticas, en estos casos, no pueden ser supervisadas cotidianamente por un agente externo al paciente $y$, al contrario, quedan bajo su directa responsabilidad; entonces, es el paciente quien en definitiva decide si cumplir o no. Por otra parte, si un paciente no cumple con las indicaciones médicas, es imposible estimar el efecto del tratamiento, lo que a su vez puede contribuir a prolongar la terapia y perpetuar enfermedades o problemas de salud e incluso conducir a la muerte, con el consecuente costo económico para el Estado en materias relativas a pérdida de productividad y costos asociados a rehabilitación (Hodgson, 2001).

Se podría afirmar que los problemas de la adherencia terapéutica han sido insuficientemente atendidos; se realizan pocas investigaciones y escasas intervenciones de modo directo y sistemático en este asunto (OMS, 2004). Por ello, se requieren trabajos investigativos que permitan estimar las tasas de adherencia terapéutica en diferentes enfermedades y grupos poblacionales, que aporten evidencias científicamente fundamentadas acerca de las causas del incumplimiento, de manera que los recursos invertidos en la salud produzcan los resultados esperados (OMS, 2004).

Esta investigación busca contribuir al cuerpo teórico en torno a la importancia de la alianza terapéutica (AT) como un factor relevante para la conformación de una buena adherencia en el área del tratamiento a las personas drogodependientes. Se espera contribuir a mejorar significativamente el tratamiento para la drogodependencia y, por ende, contribuir a la reducción de costo y fundamentalmente, a reducir el daño físico, psicológico y social al que se ven expuestas las personas que sufren esta enfermedad denominada adicción. 
En este sentido, los usuarios que acuden a los centros de tratamiento, es muy posible que se encuentren en diferentes etapas de cambio, aunque es más probable ver a usuarios en una fase de pre-contemplación; sin embargo, muchos llegan en una fase de contemplación o de preparación (Prochaska, 1999). Cabe señalar que sin importar la fase en que éstos se encuentren, el trabajo del terapeuta y de los profesionales del centro de tratamiento radica, en estas primeras etapas, en la creación del vínculo, lo que va a resultar vital para que los usuarios puedan concluir un proceso de tratamiento y lograr el cambio deseado.

A partir de la necesidad que surge en muchos terapeutas e investigadores de una conceptualización comprehensiva de la relación terapéutica, en 1975 Bordin presenta una reformulación transteórica del concepto psicoanalítico de alianza de trabajo, planteamiento que se establece sobre el trabajo de Greenson (1967), pero rompiendo con la idea de una alianza que existiría en asociación o como complemento de la neurosis de transferencia (Bordin 1975, 1976, 1980, en Santibáñez, 2003).

Bordin propone un modelo genérico de Alianza de Trabajo ${ }^{3}$ que incluye características comunes de diferentes escuelas psicoterapéuticas; en otras palabras, plantea un marco de referencia dentro del cual pueden describirse diversos tipos de terapia sin sufrir modificaciones y, al mismo tiempo, compararse sistemáticamente (Weinstein, 1997).

Bordin (1976, en Santibáñez, 2001), sugiere que la alianza sería, principalmente, una relación consciente en el aquí y el ahora, pero haciendo notar que en las fases tempranas de la terapia, ella podía ser influenciada por relaciones pasadas y vínculos no resueltos del paciente. Para él, la alianza involucra acuerdos y colaboración entre terapeuta y paciente, señalando las diferencias entre las proyecciones inconscientes de éste (transferencia) y la unión positiva paciente-terapeuta, la que se origina sobre la base de un enemigo común: el dolor y las conductas autodestructivas de quien solicita ayuda.

Según Corbella y Botella (2003), Bordin ayudó a establecer una conceptualización que aclaró hasta cierto punto las dudas sobre el papel de la transferencia y posibilitó una definición de alianza de modo que las principales escuelas terapéuticas se sintiesen cómodas con un concepto común a todas, aunque la diversidad de matices en la conceptualización de alianza terapéutica según cada perspectiva teórica continúa siendo debatida. 
Bordin (1976, en Santibáñez, 2003) percibe la alianza como una relación integrada y plantea tres componentes constitutivos de ésta: vínculo, tareas y metas, los que en combinación definen la calidad y fuerza de toda alianza. Estos componentes fueron refinados y clarificados posteriormente por el mismo Bordin (1994), entendiéndose como: Vínculo, la compleja red de conexiones entre el paciente y el terapeuta que incluyen la mutua confianza y aceptación (Horvath y Luborsky, 1993). La calidad de éste determina el tono emocional de la vivencia que el paciente tiene del terapeuta, que influye en su colaboración en el proceso terapéutico (Feixas y Miró, 1993). Tareas, son las actividades específicas (sean explícitas o implícitas) que se requiere que el paciente realice para beneficiarse del tratamiento (Safran y Seagal, 1994). Se refiere al acuerdo acerca de los medios que son adecuados para conseguir los objetivos propuestos. Son las conductas y cogniciones dentro de la terapia y que forman la sustancia de dicho proceso. En una relación de buen funcionamiento, ambas personas deberían percibir estas tareas como relevantes y eficaces y aceptar la responsabilidad para realizar estos actos (Horvath y Luborsky, 1993). Finalmente, las Metas, son los objetivos generales hacia los que se dirige la psicoterapia (Safran y Seagal, 1994). Es el grado de acuerdo entre las partes respecto a los objetivos de la intervención. Una fuerte alianza terapéutica estaría caracterizada por un terapeuta y un paciente que mutuamente respaldan y valoran las metas establecidas (Horvath y Luborsky, 1993). Estas dimensiones se influyen mutuamente y de manera continua (Safran y Muran, 2000, en Santibáñez, 2001).

Estos conceptos requieren de la colaboración entre el paciente y el terapeuta y dependen del nivel de concordancia y conexión entre ellos. Bordin (1980, en Santibáñez, 2003), manifiesta que la reciprocidad en la alianza terapéutica es el ingrediente esencial de la efectividad terapéutica. A diferencia de la perspectiva más tradicional, que examina las características del paciente y del terapeuta de modo independiente, esta noción otorga un marco dentro del cual pueden ser examinadas las variables relacionales.

Si bien para este autor el desarrollo de una adecuada alianza terapéutica es un factor de cambio esencial en todas las formas de psicoterapia, aunque su naturaleza particular varíe según la mo- 
dalidad de la terapia (Bordin, 1979, en Feixas y Miró, 1993); sostiene que una alianza positiva no es curativa por sí misma, sino que es el ingrediente que posibilita la aceptación y seguimiento del tratamiento por parte del paciente (Bordin, 1980, citado en Horvath y Luborsky, 1993).

Bordin enfatizó que, más que el método particular de tratamiento elegido, es el esfuerzo colaborativo entre terapeuta y paciente lo que puede contribuir más significativamente a la efectividad de la psicoterapia. En 1976, Bordin afirmó que la efectividad de cualquier técnica terapéutica dependerá de la claridad con que el terapeuta exponga los procedimientos técnicos, con las sensaciones que el paciente tenga de sus dificultades y las ideas del modo en que desea cambiar. Antes de que los pacientes modifiquen estas normas básicas, deben adquirir un sentido de confianza y seguridad del vínculo de la terapia.

Los planteamientos de Bordin ofrecen una alternativa al concepto dicotómico de los procesos y aspectos técnicos en la terapia al sugerir que ambos son interdependientes y que el desarrollo positivo en cada caso proporciona una base adecuada y necesaria para el crecimiento del conjunto. Este aspecto sinérgico del modelo implica que el paciente crea vínculos con el terapeuta, en parte, de acuerdo a la importancia y trascendencia que le atribuya a las intervenciones ofrecidas (Horvath y Luborsky, 1993).

Bordin trabajó con el propósito de establecer un concepto teórico general de psicoterapia, en el cual la alianza terapéutica entrega las herramientas que permiten efectuar cambios de todo tipo en relaciones de apoyo psicológico. Inicialmente se refirió a la alianza terapéutica como una condición necesaria, pero no suficiente para la psicoterapia. Posteriormente, sugirió que las diferencias en el logro alcanzado a través de distintas terapias no radican en los diferentes tipos o estilos de intervención, sino en la fuerza del vínculo terapéutico. Es éste, precisamente, el punto que la presente investigación busca destacar y enfatizar, que sin importar las bondades del modelo o de las técnicas a utilizar, si no existe una efectiva alianza terapéutica, será muy cuesta arriba para los usuarios, seguir con el proceso terapéutico y muchos de estos terminarán desertando (Horvath y Luborsky, 1993). 


\section{Material y Método}

La investigación está basada en el paradigma cuantitativo. Constituye un estudio descriptivo-correlacional. Las variables que se midieron fueron: Alianza terapéutica, adherencia al tratamiento de drogodependientes, modalidad terapéutica, así como factores sociales y económicos del paciente y del terapeuta que pueden estar influyendo en la relación terapeuta-paciente y posteriormente en la adherencia al tratamiento.

El foco de la investigación estuvo dado por la Alianza Terapéutica que se origina en la relación entre el terapeuta y los usuarios de los centros de atención a drogodependientes, del Servicio de Salud Concepción y Servicio de Salud Talcahuano, pertenecientes a la Provincia de Concepción, Región del Biobío.

Se entrevistó a la totalidad de los usuarios que cumplían requisitos de antigüedad en el tratamiento (mínimo dos meses), que fueran mayores de 18 y menores de 40 años. A su vez, se entrevistó a los terapeutas responsables del tratamiento de éstos, solicitándose la colaboración para participar entregando la información acerca del propósito de esta investigación ${ }^{4}$. Los instrumentos fueron aplicados durante el segundo semestre de 2011 a los usuarios que se encontraban en tratamiento, independiente del número de reingreso que ellos presentaran, los que representó un total de 54 usuarios que aceptaron responder y 20 profesionales terapeutas.

\section{Instrumentos aplicados:}

- Para medir Alianza Terapéutica, se usó el Inventario de Alianza Trabajo (forma T y P) de Santibáñez (2001), que es la traducción y estandarización del Working Alliance Inventory (WAI) de A. Horvath, sustentado en la teoría de Bordin sobre el vínculo de trabajo. Mide especialmente aspectos relacionados con el Vínculo, las Tareas y las Metas que son acordadas en la terapia. En lo referido a la estandarización, se logra una adaptación cultural del instrumento y presenta un lenguaje adecuado a la realidad chilena; la forma de corrección del instrumento es clara y ofrece información pertinente con el

Se entregó consentimiento informado. 
objeto de estudio; consta de 36 preguntas en un formato de escala Likert. Este inventario cuenta con una versión para el terapeuta y otra para el paciente; ambas fueron validadas y adaptadas para Chile. En su adaptación a Chile, el IAT mostró una adecuada confiabilidad en ambas versiones, con valores de .91 en la versión del paciente y .93 en la del terapeuta, y una validez convergente, con valores moderados entre $.19 \mathrm{y}$ .44 (Santibáñez, 2001).

- La Adherencia Terapéutica es entendida como el grado en que el comportamiento de una persona (tomar el medicamento, seguir el tratamiento, acudir a la citas, permanecer en rehabilitación y ejecutar cambios del modo de vida) se corresponde con las recomendaciones acordadas de un prestador de asistencia sanitaria (OMS, 2004). Esta medición se realizó por medio de la información suministrada por los centros de atención, de acuerdo a la asistencia de los usuarios y al cumplimiento de las recomendaciones e indicaciones (abstinencia, reducción del consumo).

- Las variables demográficas, sociales y de tratamiento, se midieron a través de un Cuestionario de preguntas, diseñado por el investigador.

\section{Resultados}

\section{- Alianza Terapéutica por sub-escalas, en usuarios y profesionales en tratamiento de drogodependencia}

Las respuestas de los usuarios en el inventario de Alianza Terapéutica, medido de forma global, arrojaron puntajes entre $4 \mathrm{y}$ 7 en una escala de 1 a 7 , tendencia en las puntuaciones que da como resultado un promedio total de 6.076 y DS=1.26, así también en el caso de los profesionales tratantes, cuyos promedios resultan levemente más elevados que los usuarios ( $X=6.249$, $\mathrm{DS}=0.93)$.

A nivel de cada sub-escala del inventario de alianza terapéutica, en las "metas terapéuticas" que se acuerdan en el tratamiento, los usuarios puntúan en promedio 5.826 (DS=1.386) y los tera- 
peutas promedian un total de 6,077 puntos de un máximo de 7 (DS $=0.987$ ). Cabe señalar que en ambos, usuario y terapeuta, las puntaciones más bajas se concentraron en el ítem "Desearía que..... y yo pudiéramos clarificar el objetivo de nuestras sesiones". Sin embargo, fueron los usuarios los que más bajo promediaron en este ítem ( $X=3,96$ vs. $X=5,11$ ), a su vez, son los que más perciben confusión en el proceso terapéutico. También usuarios y profesionales coincidieron en que ambos habían llegado a un buen acuerdo sobre el tipo de cambio que sería bueno para el usuario, "Creo que hemos establecido un buen acuerdo sobre cuál es el tipo de cambios que serían buenos para mî", cuya media fue muy próxima a 6,3 en ambos casos.

Por otra parte, los ítems que también presentan diferencias en los promedios, son: "Estoy en desacuerdo con acerca de lo que yo debería lograr en terapia" (X=5,48 vs. 6,03) y "Me da la impresión que y yo tenemos ideas diferentes acerca de cuáles son mis problemas" (X=4,75 vs. 5,72), "No sé qué resultados esperar de mi psicoterapia" $(X=5,33$ vs. 6,05). Lo anterior da cuenta que, si bien hay aspectos concordantes, también hay una parte de éstos (un tercio) que presentan diferencias cercanas a un punto -en escala de 1 a 7 - respecto de lo que son las metas terapéuticas establecidas en el tratamiento.

Por otra parte, con relación a la sub-escala Tareas, tanto los usuarios como los profesionales promediaron 6,060 (DS=1,23) y 6,202 (DS=0,98) puntos respectivamente, lo cual es un indicador de la baja dispersión en las repuestas de ambos. Otro de los aspectos a destacar es la similitud en las medias que presenta tanto el usuario como el profesional en el ítem que aborda percepciones en torno a aspectos de las tareas terapéuticas, como son los acuerdos que se establecen en terapia, lo que el proceso terapéutico aporta en la comprensión del problema y sobre lo que se espera del usuario en las sesiones. Así también, los ítems con mayor diferencia en los promedios, aunque ésta no supera el 0,5 punto, corresponden a "Encuentro confuso lo que estoy haciendo en terapia", "Creo que el tiempo que...... y yo estamos juntos en la(s) sesión(es) no es aprovechado de modo eficiente", "Encuentro que lo que....... y yo hacemos en terapia no se relaciona con mis problemas actuales", "Estoy frustrado(a) por las cosas que estoy haciendo en terapia", "Siento que las cosas que ........ me pide que haga, no tienen sentido". 
En los referido a la percepción del Vínculo, sub-escala con mayor convergencia, ambos tendieron a dar repuestas elevadas, con puntuaciones muy similares, promedios de 6,341 (DS $=1.15)$ para los usuarios y de 6,34 (DS=0.81) para los profesionales: "Piensan que se entienden en la relación", "Creen que se estiman", "Que se respetan mutuamente", entre otros; los aspectos que presentan una mayor diferencia corresponden solo a dos casos "Siento que...... no es completamente sincero(a) en sus sentimientos hacia mi" (X=5,537 vs. $X=6,667)$ y "Mi relación con .... es muy importante para mi" (X=6,51 vs. $\mathrm{X}=6,07)$; el primero de estos indicadores arroja interrogantes respecto de las respuestas a los ítems que consultan por sentimientos de estima, respeto y preocupación por el otro.

Para establecer si existen diferencias significativas entre las puntuaciones totales de la Alianza terapéutica, de acuerdo a las respuestas del profesional y del usuario, se utilizó el proceso estadístico “T de Student" para grupos pareados. Por una parte, los resultados arrojados por este procedimiento indican que no existen diferencias significativas en los puntajes totales de la alianza terapéutica entre el profesional y el usuario, es decir, ambos valores convergen entre sí y la relación entre éstos es positiva ( $p=0.07$ ), situación que se repite para las sub-escalas Tareas y de Vínculo. Ambas pruebas superaron los 0.05, por lo que se rechaza la hipótesis de diferencia de medias; en este sentido, las precepciones que poseen los profesionales y los usuarios no presentan diferencias significativas, lo cual permite afirmar que ambos tienen opiniones muy similares en torno al Vínculo que han creado y sobre las estrategias que se utilizan en las terapias (Tareas).

Por otra parte, sí es posible evidenciar diferencias significativas en la puntuación del profesional y del usuario en la sub-escala "Metas Terapéuticas" ( $\mathrm{T}=2.31$, P $<0.02)$. El que existan diferencias en este componente de la alianza puede contribuir a debilitar la alianza terapéutica establecida entre ambos, ya que una fuerte alianza se caracteriza por un profesional y un usuario que mutuamente respaldan y valoran las metas establecidas en el tratamiento (Bordin, 1976, citado en Santibáñez, 2003). 
Tabla 1. Comparación de los resultados del inventario de alianza terapéutica, tanto del usuario y como del Profesional mediante el Test de Student para grupos pareados.

\begin{tabular}{|l|l|l|l|c|c|}
\hline Variables & N & Media & DS & T Value & Pr>P \\
\hline $\begin{array}{l}\text { Alianza Terapéutica (AT) } \\
\text { Profesional - AT Paciente }\end{array}$ & 54 & 6.2593 & 3.4474 & 1.82 & 0.0751 \\
\hline $\begin{array}{l}\text { Metas del Terapeuta - Metas } \\
\text { del Paciente }\end{array}$ & 54 & 3.0185 & 1.3073 & 2.31 & $\mathbf{0 . 0 2 4 9}$ \\
\hline $\begin{array}{l}\text { Tareas del Terapeuta - } \\
\text { Tareas del Paciente }\end{array}$ & 54 & 1.7037 & 1.3065 & 1.30 & 0.1979 \\
\hline $\begin{array}{l}\text { Vínculo del Terapeuta - } \\
\text { Vínculo del Paciente }\end{array}$ & 54 & 1.537 & 1.2776 & 1.20 & 0.2343 \\
\hline
\end{tabular}

Fuente: Tesis Magíster "Alianza Terapéutica y Adherencia a Tratamiento en drogodependencia", David Vidal C.

\section{Alianza Terapéutica y Adherencia Terapéutica}

La alianza terapéutica es significativa en relación a la adherencia al tratamiento de drogodependencia (valor $\mathrm{f}=8.01, \mathrm{P}=0.0066$ ) y explica un $13,3 \%$ de ésta. Lo anterior indica que por el aumento de un punto en la escala de Alianza Terapéutica del profesional, la adherencia del paciente aumenta en un 0,24\%. Este resultado guarda relación con anteriores investigaciones en torno al tema que evidencian que, de todas las variables estudiadas del proceso terapéutico que pudieran predecir la adherencia, el resultado más eficaz es el de la alianza terapéutica (Horvath y Symonds, 1991).

En lo referido a la percepción que tiene el profesional sobre el cumplimiento de las Tareas terapéuticas establecidas en el curso del tratamiento (Subescala Tareas), se estableció que el cumplimiento de éstas explican un 18,4\% de la adherencia al tratamiento que presenta el usuario $(p=0.0012)$, lo cual representa el nivel de explicación más alto en torno a la adherencia terapéutica. Hay que señalar que el cumplimiento de las tareas terapéuticas guarda una estrecha relación con la adherencia, ya que esta última se establece precisamente por el grado en que el comportamiento del usuario se corresponde con las recomendaciones acordadas con el profesional de la salud (OMS, 2004), por lo que a mayor cumplimiento de las tareas terapéuticas, mayor será la adherencia que presente el usuario. 
Al igual que las Tareas, la percepción que tiene el profesional del Vínculo, como un elemento aislado de la alianza, resulta ser también significativa estadísticamente $(F=3,72, P=0,05)$, y explica un $6,6 \%$.

El último componente de la alianza terapéutica son las Metas terapéuticas. Se puede observar que la percepción del profesional sobre el grado de acuerdo o desacuerdo que presenta el usuario con las metas establecidas en el tratamiento, explica el $10 \%$ de la adherencia que pueda presentar el usuario en el curso del tratamiento y es significativo a 0.02 (Tabla $\mathrm{N}^{\circ} 2$ ).

Por su parte, al analizar estadísticamente los resultados arrojados por el inventario de Alianza Terapéutica respondido por los usuarios respecto a su relación con los profesionales a cargo de su tratamiento, se puede señalar que la percepción de éstos sobre la alianza terapéutica, en este caso, no resulta ser significativa para explicar la adherencia al tratamiento (Tabla $\mathrm{N}^{\circ} 2$ ).

Tabla 2. Explicación de Alianza terapéutica del Profesional y Usuario en la Adherencia al Tratamiento de drogodependientes (regresión lineal simple).

\begin{tabular}{|l|l|l|l|l|l|}
\hline Variable & GL & $\begin{array}{l}\text { Parámetro } \\
\text { Estimado }\end{array}$ & $\begin{array}{l}\text { F } \\
\text { calculado }\end{array}$ & $\begin{array}{l}\text { Prueba } \\
\text { F }\end{array}$ & R2 \\
\hline $\begin{array}{l}\text { Alianza Terapeútica } \\
\text { Profesional }\end{array}$ & 53 & 0.24210 & 8.01 & 0.0066 & 0.1335 \\
\hline Vínculo del Profesional & 53 & 0.64065 & 3.72 & 0.0593 & 0.0667 \\
\hline Tareas del Profesional & 53 & 0.72752 & 11.79 & 0.0012 & 0.1848 \\
\hline Metas del Profesional & 53 & 0.22150 & 5.84 & 0.0192 & 0.1010 \\
\hline Alianza Terapéutica \\
usuario & 53 & -0.1753 & 0.04 & 0.8361 & 0.0008 \\
\hline Vínculo del usuario & 53 & -0.02279 & 0.01 & 0.9169 & 0.0002 \\
\hline Tareas del usuario & 53 & -0.08599 & 0.14 & 0.7053 & 0.0028 \\
\hline Metas del usuario & 53 & -0.01514 & 0.007 & 0.9461 & 0.0001 \\
\hline
\end{tabular}

Fuente: Tesis Magíster "Alianza Terapéutica y Adherencia a Tratamiento en drogodependencia”, David Vidal C. 


\section{Factores Socio-demográficos y de Salud y Adherencia Terapéutica del usuario}

Los factores sociodemográficos contemplados en la investigación no arrojaron niveles de explicación significativos en torno a la adherencia terapéutica, aunque cabe señalar que otras investigaciones sí han señalado que el sexo, la edad y el nivel académico influyen en la adherencia que presentan los usuarios (OMS, 2004).

Entre los factores que sí ofrecieron explicación sobre Adherencia Terapéutica es posible mencionar: "frecuencia del consumo" y el "tipo de droga" de preferencia del usuario. En este sentido, la frecuencia en el consumo explica un 10,4\% y el tipo de "droga de consumo de preferencia", explica un $8 \%$ de la adherencia terapéutica: Los usuarios que presentaban un consumo de "una a dos veces por semana" ( $\mathrm{P}=0.0189$ ) al entrar al tratamiento, presentan 11,36 puntos menos de adherencia que los que ingresan con una alta frecuencia de consumo; y los usuarios que consumían solo "alcohol y marihuana" (valor $\mathrm{P}=0.0441$ ), presentan 10,03 puntos menos en la adherencia, en relación con los usuarios que consumían múltiples sustancias (Tabla $\mathrm{N}^{\circ} 3$ ). En este sentido, es probable que los usuarios que presenten un bajo consumo se encuentren en una fase pre-contemplativa del cambio (Prochaska, 1999) debido al poco riesgo que perciben en su hábito; por el contrario, el usuario que ha abusado más de la sustancia puede encontrarse en estadios de cambios más avanzados debido al efecto de las drogas en múltiples áreas de su vida.

Por otra parte, al explorar la relación entre características del profesional tratante y adherencia al tratamiento, no se encontró significación estadística con ningún factor demográfico o de especialidad disciplinaria. Estos mismos resultados son compartidos por otras investigaciones (Bueno, A. et al., 2001; Sledge, W., et al., 1990) en donde se ha encontrado que factores como la edad o el tipo de formación del profesional, no explican la adherencia al tratamiento. Diferente es lo que ocurre con la experiencia del profesional expresada en años; el Profesional con más años de experiencia en el área (tres o más) obtiene mayor puntaje en adherencia de los usuarios (79,5\%) que quienes tienen un año, cuyos usuarios obtienen hasta 23 puntos menos comparativamente (Tabla N³). 
Tabla 3. Análisis de covarianza de los factores del usuario y profesional que explican la Adherencia Terapéutica.

\begin{tabular}{|c|c|c|c|c|c|c|c|}
\hline Variables & $\mathrm{N}$ & $\%$ & P. Estimado & $\mathrm{T}$ value & $\operatorname{Pr}>\mathrm{T}$ & $\operatorname{Pr}>\mathrm{F}$ & R2 \\
\hline \multicolumn{8}{|c|}{ Drogas de consumo } \\
\hline $\begin{array}{l}\text { Alcohol, } \\
\text { marihuana, } \\
\text { cocaína y } \\
\text { pasta base }\end{array}$ & 26 & 48 & 77.92308 & 31.62 & $<.0001$ & \multirow[t]{3}{*}{0.1088} & \multirow[t]{3}{*}{0.0833} \\
\hline $\begin{array}{l}\text { Alcohol, } \\
\text { marihuana y } \\
\text { cocaína }\end{array}$ & 19 & 35 & -0.39676 & -0.10 & 0.9171 & & \\
\hline $\begin{array}{l}\text { Alcohol y } \\
\text { marihuana }\end{array}$ & 9 & 17 & -10.03419 & -2.06 & 0.0441 & & \\
\hline \multicolumn{8}{|c|}{ Frecuencia de consumo } \\
\hline Cinco o más & 32 & 59 & 78.81250 & 35.89 & $<.0001$ & \multirow[t]{3}{*}{0.0605} & \multirow[t]{3}{*}{0.1042} \\
\hline Tres a cuatro & 13 & 24 & -3.35096 & -0.82 & 0.4159 & & \\
\hline Uno a dos & 9 & 17 & -11.36806 & -2.43 & 0.0189 & & \\
\hline \multicolumn{8}{|l|}{$\begin{array}{l}\text { Años de } \\
\text { Experiencia }\end{array}$} \\
\hline Tres o más & 9 & & 79.55556 & 22.36 & $<.0001$ & 0.0028 & 0.4991 \\
\hline Dos & 6 & & -4.05556 & -0.72 & 0.4808 & & \\
\hline Uno & 5 & & -23.95556 & -4.02 & 0.0009 & & \\
\hline
\end{tabular}

Fuente: Tesis Magíster "Alianza Terapéutica y Adherencia a Tratamiento en drogodependencia", David Vidal C.

\section{Conclusiones}

A partir de la investigación realizada ha sido posible profundizar en cómo la "Alianza Terapéutica" y otros factores relacionados con el tratamiento, pueden explicar parte de la variación en la adherencia a tratamiento, manifestada por el usuario drogodependiente.

\section{La percepción de la alianza terapéutica desde el usuario y el profesional}

Uno de los objetivos de esta investigación fue establecer si existían diferencias en cómo usuarios y profesionales vinculados en el tratamiento de la drogodependencias, percibían la alianza terapéutica (AT), y en cada uno de sus componentes, ya que las convergencias o divergencias que pudieran producirse entre es- 
tas variables son un indicador del éxito que pudiera tener la intervención terapéutica (Corbella \& Botella, 2003).

En este sentido, al contrastar la media de respuesta de ambos grupos (usuario y profesional) en el Inventario Alianza Terapéutica, no se pudieron establecer diferencias significativas, por lo que usuarios y profesionales percibieron, a grandes rasgos, el fenómeno de la Alianza Terapéutica de forma muy similar, incluso promediando un puntaje por respuestas muy cercano en el inventario AT. En este sentido, los profesionales arrojaban un promedio de respuesta de 6.249 y los usuarios de 6.075.

Sin embargo, al revisar cada una de las sub-escalas que componen la AT, se pudo apreciar que no siempre estas percepciones coincidieron positivamente. Un ejemplo de esto se desprende de los valores arrojados en la subescala de Metas Terapéuticas, donde la diferencia en las puntuaciones de ambos, resulta ser estadísticamente significativa (P:0.0249). Las metas terapéuticas hacen referencia a los acuerdos entre el paciente y el terapeuta respecto a los objetivos de la terapia, es decir, el mutuo consentimiento acerca de los objetivos que pretende lograr con la intervención, por lo que una fuerte alianza se caracteriza por un profesional y un usuario que mutuamente respaldan y valoran las metas establecidas en el tratamiento (Bordin, 1976, citado en Santibáñez, 2000). El que existan divergencias en relación a las metas puede alterar los resultados esperados en el tratamiento, ya que las metas terapéuticas engloban cambios profundos en el estilo de vida a largo plazo, que sirven de guía para el usuario, no solo en el curso del tratamiento, sino cuando egresa de éste (Watson y Greenberg, 1995).

Por lo anterior, se hace necesario profundizar -a futuro- en identificar las variables que pudieran explicar las divergencias que se produjeron en las Metas Terapéuticas, ya que éstas engloban cambios complejos asociados a estilos de vida, por lo que son de suma importancia para poder establecer el impacto real de los tratamientos a drogodependientes realizados en los centros de atención contemplados en este estudio.

En este sentido, cabe señalar que el promedio de meses en tratamiento del universo fue de 5,5 meses, por lo que muchos de éstos pudieran encontrarse en fases tempranas del proceso de cambio. De esta forma, encontrarse en una fase precontemplativa o con- 
templativa, propuesta por el Modelo de Cambio de Prochaska, predispone la comprensión del riesgo o los daños generados por el consumo de sustancias, por lo que tanto las metas como la abstinencia total, promovida por la mayoría de los centros de tratamientos estudiados, encuentra resistencia en estos usuarios y genera estas divergencias en las metas a corto o largo plazo propuestas por los terapeutas o profesionales del centro.

Es importante destacar que, aunque existan diferencias en la percepción de las metas establecidas en el tratamiento, a nivel general de la Alianza terapéutica como tal y de los otros dos componentes de ésta -el Vínculo Terapéutico y las Tareas Terapéuticas-, hay una valoración positiva tanto por parte del profesional como del usuario de la Alianza Terapéutica que se ha formado entre ambos, lo cual permitiría sostener y facilitar -en el tiempo- el proceso terapéutico e influir en el resultado final de dicho tratamiento.

\section{Adherencia Terapéutica y Alianza Terapéutica}

La adherencia Terapéutica, como hemos reiterado, hace referencia al grado en que el comportamiento de una persona (tomar el medicamento, seguir un régimen alimentario y ejecutar cambios del modo de vida) se corresponde con las recomendaciones acordadas de un prestador de asistencia sanitaria (OMS, 2004). La adherencia requiere la conformidad del usuario respecto de las recomendaciones. Esta nueva concepción del tratamiento promueve que este usuario deba ser socio activo con los profesionales de la salud en su propia atención y esa buena comunicación entre ambos es un requisito esencial para una práctica clínica efectiva.

Una de las áreas que ha retomado mayor relevancia en la evaluación de la estructura del sistema de salud, es la que se refiere a la relación terapeuta-paciente y su relación con la adherencia terapéutica. Existen investigaciones donde se confirma (Sarro, 2003), por ejemplo: a) Que a mayor satisfacción del paciente en la relación con su terapeuta, habrá un mayor y mejor cumplimiento de los programas de tratamiento. b) Que a mayor satisfacción en el paciente, habrá menor probabilidad de deserción. c) Que a una mayor duración, es decir, continuidad en la relación, mejor será el cumplimiento. 
La adherencia terapéutica en el área del tratamiento de la drogodependencia ha sido relacionada, por varios enfoques, como un factor determinante para el éxito o mantenimiento del tratamiento. Esta relación entre la duración de un tratamiento y el posterior cumplimento de la indicaciones, ha recibido especial atención en el marco del tratamiento de las adicciones. Vaillant (1966) fue uno de los primeros que contempló entre sus análisis esta relación y fue precisamente él quien sugirió la existencia de una relación inversa entre la duración de la estancia de los pacientes con adicciones en el Hospital Lexington de Nueva York y el número de recaídas que el sujeto puede experimentar al término del tratamiento. Rivera (2001) ha establecido que la tasa más alta de abandonos se da en los primeros 30 días, continúa creciendo hasta los 3 meses y se empieza a reducir poco a poco a partir de ese periodo; la probabilidad de una permanencia continuada en el programa aumenta considerablemente después de 90 días, por lo que la probabilidad de abandono va a ir decreciendo con el paso del tiempo.

Esta investigación estaba orientada a establecer el nivel de explicación que pudiera arrojar la alianza terapéutica en relación a la adherencia al tratamiento que presentaron los usuarios. Desde esta perspectiva, el nivel de explicación de la AT es de un 13,35\% de acuerdo a la prueba de análisis de covariancia. Este nivel de explicación no es menor, teniendo en cuenta las múltiples variables que pueden influir en la adherencia que presenten los usuarios, por tanto, lograr este porcentaje explicado por la AT permite entender la importancia de la AT en el curso de un tratamiento y acercarnos más a la comprensión del fenómeno de la adherencia.

Respecto a los componentes del Inventario de AT: el Vínculo, las Tareas y las Metas terapéuticas, todos arrojaron un nivel de explicación significativo en relación a la adherencia; sin embargo, la dimensión Tareas aportó el nivel de explicación más alto, con un $18,48 \%$. Por lo anterior resulta fundamental reconocer cómo se perciben las actividades a realizar, las cuales están intrínsecamente relacionadas con la intervención que se esté realizando, ya que el cumplimento de éstas incide directamente en el aumento o no de la habilidades requeridas para enfrentar los obstáculos del tratamiento (Bordin, 1976). Teniendo en cuenta que la adherencia terapéutica es precisamente el grado 
en que el comportamiento de una persona -tomar el medicamento, seguir un régimen alimentario y ejecutar cambios del modo de vida- se corresponde con las recomendaciones acordadas de un prestador de asistencia sanitaria (OMS 2004), el componte de la Tareas terapéuticas resulta ser un buen predictor de la adherencia terapéutica y del posterior éxito de la intervención.

Por ello, resulta importante promover dentro de las políticas de salud, principalmente las relacionadas con la atención de usuarios con problemas de drogodependencia, la relevancia que tiene la alianza terapéutica para explicar el fenómeno de la adherencia terapéutica. Debemos recordar que la adherencia terapéutica deficiente es la principal razón de complicaciones médicas y psicosociales de la enfermedad (OMS, 2004), ya que reduce la calidad de vida de los pacientes y aumenta la complejidad de la intervención, por cuanto una adherencia deficiente produce recaídas más intensas, aumento de la cronicidad de la adicción e incrementa el riesgo de dependencia a medicamentos utilizados para controlar la ansiedad o el deseo de consumo (Rivera, 2001).

Las intervenciones que puedan eliminar las barreras a la adherencia terapéutica, como es el caso del fomento entre todos los profesionales del área de la drogodependencia (trabajadores sociales, psicólogos y psiquiatras principalmente) de los beneficios de una fuerte alianza Terapéutica, debiera convertirse en un componente central de los esfuerzos para mejorar las políticas de salud. Aumentar la calidad de la Alianza terapéutica en las intervenciones quizás tenga una repercusión mucho mayor sobre la salud de los usuarios que cualquier mejora que pudiesen realizar los centros de atención a drogodependientes.

Sin embargo, esta visión sobre la importancia de la AT en el tratamiento, todavía no ha tenido relevancia dentro de las políticas públicas en Chile. La Estrategia Nacional de Drogas 2011-2014 centra su esfuerzo en el aumento de cupos de tratamiento, dejando de lado la importancia de mejorar la calidad de la atención, así como la importación de la relación terapéutica. La calidad de la alianza es el predictor más fuerte en el éxito de los tratamientos (Orlinsky, Grawe y Parks, 1994). 


\section{Adherencia Terapéutica y otros Factores asociados}

Esta investigación analizó una diversidad de factores que pudiesen arrojar niveles de explicación en relación a la AT, sin embargo, solo podemos destacar la variable relacionada con la frecuencia de consumo, como la única que pudo ofrecer un nivel de explicación significativo sobre el fenómeno de la adherencia terapéutica, además de la Alianza Terapéutica, en usuarios de los centros analizados.

En este sentido, el nivel de explicación de esta variable es de $10,41 \%$, siendo los que presentaban menor consumo los que menor adherencia al tratamiento registraban. Algunos investigadores han afirmado que una de las razones que impulsa el cambio o la búsqueda de tratamiento es precisamente cuando el usuario ha abusado de drogas duras y de forma múltiple, ya que este tipo de consumo provoca un deterioro tanto en la salud del usuario como en otras esferas de su vida, lo que impulsa al sujeto a buscar ayuda y adherirse al tratamiento (Caraballo $\mathcal{E}$ colaboradores, 2007).

Las explicaciones que se ofrecen desde el punto de vista teórico argumentan que la frecuencia de consumo influye sobre la percepción que se tiene en torno al daño que pudiera producir la sustancia (CONACE, 2006), porque usuarios que ingresan al centro de tratamiento con una frecuencia baja del consumo, pueden sentir que ejercen mejor control sobre el uso de la sustancia, por lo que no sentirían la necesidad de asistir a tratamiento, lo que a futuro influiría negativamente en su salud y su vida.

Por otra parte, las variables vinculadas al profesional y que mejor explicación ofrecieron en relación a la adherencia del usuario, fueron la experiencia y el nivel de especialización de éste: a menor experiencia del profesional, menor será la adherencia del usuario. Cabe destacar que dicho factor ha sido considerado como importante por la organización Mundial de la Salud, destacando que la poca experiencia o especialización del equipo influye negativamente en el tratamiento del usuario (OMS, 2004).

\section{Bibliografía}

Altamura, A. E Malhi, G. (2000). Compliance on mayor psychoses. International Journal of Psychology and Clinical Practice, 4, pp. 81-82. 
Bachman R. (1993). Better compliance: physicians making it happen. Am Fam Physician. Boston, EE.UU.

Bordin, E. S. (1976). The generalization of the psychoanalytic concept of the working alliance. Psychotherapy: Theory, Reseach and Practice, 16, pp. 252-260.

Bordin, E. S. (1994). Theory and research on the therapeutic working alliance: New directions. En A. O. Horvath y L. S. Greenberg (Eds.). The working alliance: Theory, research, and practice. New York: Wiley.

Bueno, A.; Córdoba, JA.; Escolar, A.; Carmona, J. E Rodríguez, C. (2001). El abandono terapéutico. Actas Españolas de Psiquiatría. España.

CONACE (2006). Estudio Nacional sobre costos humanos, sociales y económicos de la drogas en Chile. Santiago de Chile. Recuperado de: http://www.senda.gob. cl/wp-content/uploads/2011/04/2006_Costos_ Alcohol_Drogas.pdf

CONACE (2010). Noveno Estudio Nacional de Drogas en Población General de Chile, 2010. Santiago de Chile. Recuperado de: http://www.senda.gob.cl/ wp-content/uploads/2012/02/2010_noveno_ estudio_nacional.pdf

Corbella, S. y Botella, L. (2003). La alianza terapéutica: historia, investigación y evaluación [versión electrónica]. Revista Anales de Psicología, 2 (19), pp. 205 - 221. Recuperado de: http://www.um.es/analesps/v19/ v19_2/04-19_2.pdf

Feixas, G. y Miró, M. (1993). Aproximaciones a la psicoterapia. Barcelona: Editorial Paidós.

Hartley, D. (1985). Research on the therapeutic alliance in psychotherapy. En R. Hales \& A. Frances (Eds.), Psychiatry update: American Psychiatric Association annual review (pp. 532-549). Washington DC: American Psychiatric Association Press.

Hernández, JL.; Acosta, F.; Pereira, J. (2009). Adherencia terapéutica. Generalidades. En Hernández, JL. (Compilador). Adherencia Terapéutica. Cuadernos de Psiquiatría comunitaria Vol. 9, $\mathrm{N}^{\circ}$ 1, pp. 13-28. Recuperado de: http://www.aen.es/docs/cuadernos9-1.pdf

Hodgson, T. (2001). Medical care expenditures for hypertension, its complications, and its comorbidities. Medical 
Care. Recuperado de: http://www.ncbi.nlm.nih. gov/pubmed/1 1404643

Horvarth, A.O. y Luborsky, L. (1993). The role of the therapeutic alliance in psychotherapy. Journal of Consulting and Clinical Pychology, 51(4), pp. 561-573.

Horvath, A. O. E Symonds, B. D. (1991). Relation between the working alliance and outcome in psychotherapy: A metaanalysis. Journal of Counseling Psychology, 38, 139149.

OMS; 2004. Adherencia a los tratamientos a largo plazo. Pruebas para la acción. Ginebra, Suiza. Recuperado de: http:// www.paho.org/hq/index.php?option=com_ docmanEtask=doc_viewEgid=18722EItemid=

Orlinsky, D.; Grawe, K. E Parks, K. (1994). Process and outcome in psychotherapy. En A.E. Bergin E S.L. Garfield (Eds.), Handbook of psychotherapy and behavior change (4ta. ed., pp. 270-378). Nueva York: Wiley.

Pereira, J.; Acosta, J.; Hernández, JL. (2009). La Adherencia en psicoterapia. En Hernández, JL. (Compilador). Adherencia Terapéutica. Cuadernos de Psiquiatría comunitaria Vol. 9, N 1, pp. 67-78. Recuperado de: http://www.aen.es/docs/cuadernos9-1.pdf

Prochaska, J. (1999). How Do People Change, and How Can We Change to Help Many Mor e People? In: M. Hubble, B. Duncan and S. Miller (Eds.) The Heart and Soul of Change: What Works in Therapy, Washington, D.C: American Psychological Association.

Planes, M. (mayo 1991). La influencia de diversos tipos de expectativa en el cumplimiento terapéutico. Comunicación presentada al I1 Congrés International (Latini Dies), Sitges.

Rivera, P.(2001).Estadiosy procesos decambioen drogodependientes en tratamiento ambulatorio. Adicciones Vol. 13. $\mathrm{N}^{\circ} 2$, pp. 147-152.

Safran, J. y Segal, Z. (1994). El proceso interpersonal en la terapia cognitiva. Barcelona: Editorial Paidós.

Santibáñez, P. (2001). La alianza terapéutica en psicoterapia: traducción, adaptación y examen inicial de las propiedades psicométricas del Working Alliance Inventory en Chile. Tesis para optar al grado de Magíster en Psicología, Mención Psicología Clínica. Santiago de Chile: Pontificia Universidad Católica. 
Santibáñez, P. (2003). La alianza terapéutica en psicoterapia: el "inventario de alianza de trabajo" en Chile [Versión electrónica]. Revista Psykhe, 1 (12). http:// www.ocenet.oceano.com/Salud/viewSimple.do

Sarró, S. y Pomarol, E. (2003). Factores que influyen en el cumplimiento terapéutico. En J.M. Costa Molinari (Coord.): Área de conocimiento 12. Barcelona: Universidad Autónoma de Barcelona.

Castro, A. (2001). ¿Son eficaces las psicoterapias psicológicas? Rev. Psicología, cultura y sociedad. Recuperado de: http://www.palermo.edu/cienciassociales/ publicaciones/pdf/Psico3/3Psico\%2005.pdf

Sledge, W.; Moras, K.; Hartley, D.; Levine, M. (1990). Effect of TimeLimited Psychoterapy on Patient Dropout Rates. Am J Psychiatry 1990;147, pp. 1341-1347.

Vaillant,G.E. (1966). A 12-year follow-up of New York narcotic addicts III. Archives of General Psychiatry, 15, pp. 599-609.

Watson, J. C. y Greenberg, L. S. (1995). Alliance ruptures and repairs in experiential therapy. In Session: Psychotherapy in Practice, 1 (1), pp. 19-32.

Weinstein, X. (1997). Variables del paciente que influyen sobre la alianza terapéutica. Memoria para optar al título de Psicóloga, Escuela de Psicología. Santiago, Chile: Universidad de Chile. 